\title{
INCREASED INCIDENCE OF FUNGAL SINUSITIS IN COASTAL REGION: A
}

\section{CASE SERIES}

\author{
I. Arumugam¹, Jalagandeesh B², Ganesh Bala³, Ishani Borah, Sandeep Jith ${ }^{5}$
}

\section{HOW TO CITE THIS ARTICLE:}

I. Arumugam, Jalagandeesh B, Ganesh Bala, Ishani Borah, Sandeep Jith. "Increased Incidence of Fungal Sinusitis in Coastal Region: A Case Series". Journal of Evolution of Medical and Dental Sciences 2015; Vol. 4, Issue 81, October 08; Page: 14240-14244, DOI: 10.14260/jemds/2015/2024

\begin{abstract}
Many fungal species cause infection in the nose and paranasal sinuses. Almost half of them are capable of causing systemic and CNS infections. Clinical presentation, imaging features, and treatment differ based on type of fungal sinusitis. We retrospectively reviewed 40 patients admitted and treated surgically for sinusitis in otorhinolaryngology department of the Vinayaka Missions Medical College \& Hospitals, Karaikal for the last three months, April, May \& June 2015. The clinical presentation, laboratory examination, radiological finding, cultre report and treatment method were analyzed. We had encountered 11 cases of fungal sinusitis, which is very much a high value of incidence than reported elsewhere (27.5\%). A combined prospective and retrospective study is currently going on in our department to determine the etiology for such increased incidence. We are hereby discussing the classification of fungal sinusitis and present 4 cases of each type.
\end{abstract}

KEYWORDS: Fungal sinusitis, Mycetoma, Allergic mucin, Immunocompetent, Coastal region.

INTRODUCTION: The paranasal sinuses are a group of paired air containing spaces that surround the nasal cavity extending superiorly to the skull base and laterally to encompass the medial wall and floor of the orbit. They include the maxillary, ethmoidal, frontal, and sphenoidal sinuses with each having narrow ostium that opens into the nose. ${ }^{1}$

Inflammation of their mucosal lining is referred to as sinusitis. This is a frequent occurrence and their complications, which can be life threatening, have become rare especially in the developed nations since the introduction of antibiotics. ${ }^{2}$

MATERIALS AND METHODS: We retrospectively reviewed 40 patients admitted and treated surgically for sinusitis in otorhinolaryngology department of the Vinayaka Missions Medical College \& Hospitals, Karaikal for the last three months, April, May \& June 2015. The clinical presentation, laboratory examination, radiological finding, bacteriological report and treatment method were analyzed.

40 patients were admitted and treated surgically for sinusitis. The mean age was 40-60 years. There was female preponderance. The most common infection was of the maxillary sinus. The culture report of the exudate showed Staphycoccus aureus in 16 patients as the most common pathogen followed by B Hemolytic Streptococci, mycobacterium tuberculosis. We had encountered 11 cases of fungal sinusitis, which is very much a high value of incidence than reported elsewhere (27.5\%). We are hereby discussing the classification of fungal sinusitis and present 4 cases of each type, we encountered.

DISCUSSION: The most accepted classification of fungal sinusitis is represented by the system proposed by deShazoet al. ${ }^{3}$ Fungal rhinosinusitis presents in five clinico-pathologic forms, each with distinctdiagnostic criteria, treatment and prognosis. 
The invasive forms are acute fulminant, chronic and granulomatous invasive fungal sinusitis. The noninvasive forms are fungal ball ("Sinus mycetoma") and allergic fungal sinusitis (AFS).

Acute Invasive Fungal Sinusitis: It is the most lethal form, with mortality of about $50-80 \%$. It occurs in immunocompromised patients (AIDS, haematologic diseases, type 1 diabetes mellitus) with a fatal outcome in the absence of treatment. ${ }^{4}$ the most frequent sites of involvement, are near the middle turbinate, the septum and more rarely the inferior turbinate. ${ }^{5}$

Case 1: A 58 year old female, a known diabetic patient came with complaints of left sided nasal obstruction and blood mixed discharge from left. Anteriorrhinoscopy showed black necrotic masses in the left middle turbinate and meatus blocking the entire nostril. CT scan revealed left maxillary sinusitis with hyperdense foci with sclerosis and erosion of the maxillary sinus and inferior orbital wall. Apart of the mass was removed endoscopicaly and send for HPE and culture which revealed growth of Mucorales. Radical surgical debridement was done and she was treated with IV Amphotrecin B.

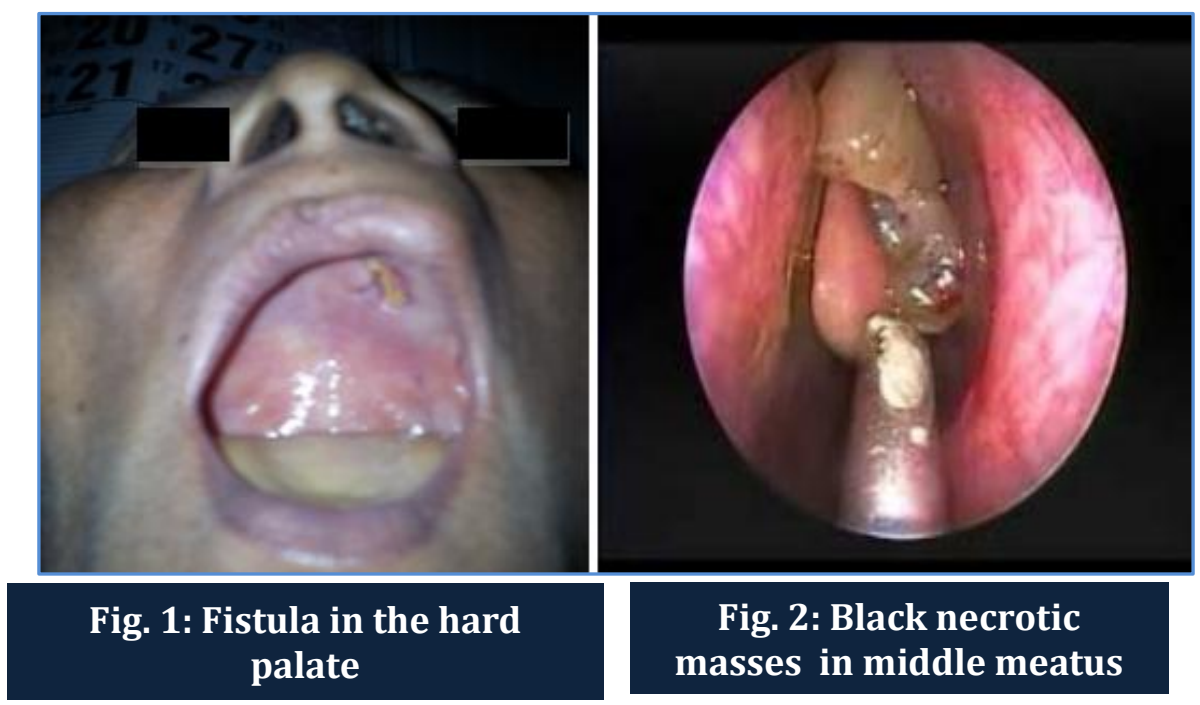

Chronic Invasive Fungal Sinusitis: Invasive fungal rhinosinusitis is probably one of the less frequent forms of fungal rhinosinusitis, most of them being reported in northern Africa and Asia. ${ }^{6}$ Two fonns are usually described: granulomatous and non-granulomatous, based on the presence or absence of granulomas within tissue. ${ }^{3}$ It occurs in healthy individuals, sometimes after a previous history of chronic rhinosinusitis. Progression is over months to years with fungal organisms invading mucosa, submucosa, blood vessels, muscles and bony walls.

Case 2: A 47 year old female came with complaints severe headache and blurring of vision and swelling around the left eye for 4 months. She had history of chronic headache and bilateral nasal obstruction which she neglected for the past 7 years. Anteriorrhinoscopy showed greyish white mass completely filling the left nasal cavity. She has history of diabetes and she is under medication for the last 4years.CT Scan reveals hyperdense mass filling left nasal cavity, maxillary, ethmoid sinuses and invading the roof of the orbit on the left side, involving the extraoccular muscles, pushing the left eyeball inferiorly. Intraoperatively greenish brown mucopurulent debris was removed from the 


\section{CASE REPORT}

maxillary sinus, ethmoidal cells and from the roof of the orbit. Post operatively significant improvement in visual acuity was seen.

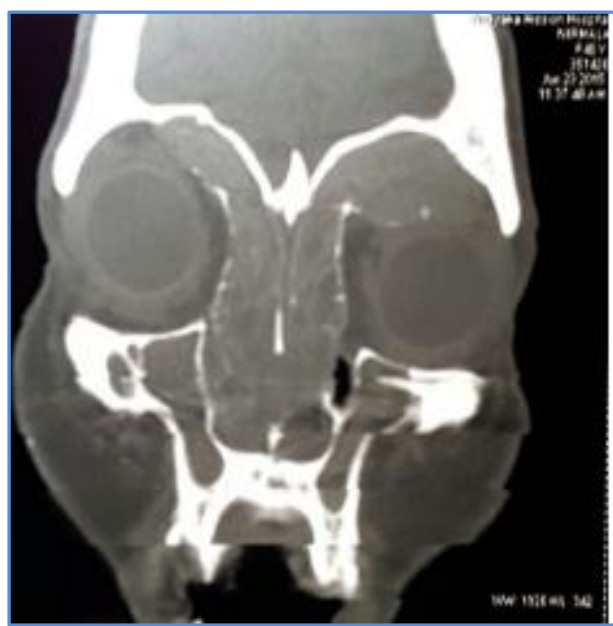

Fig. 3: Pre-operative

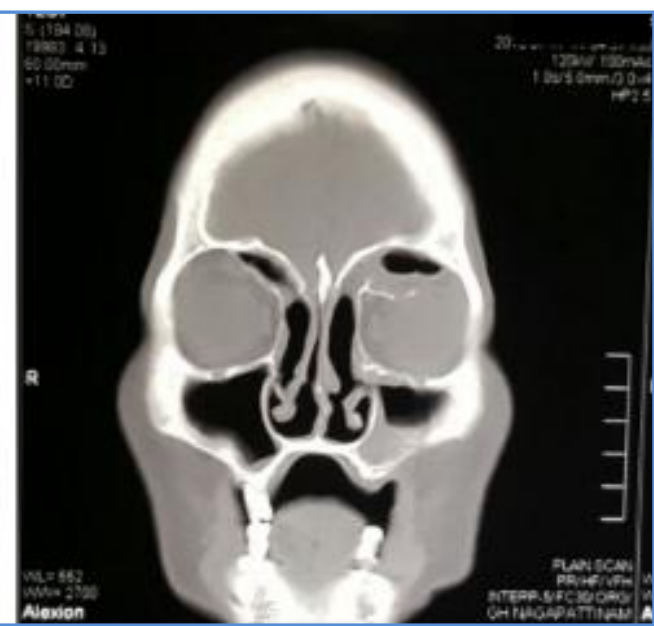

Fig. 4: Post-operative

Fungal Ball (Mycetoma): The fungus ball is now well defined as the presence of tangled mats of hyphae in one or more sinus cavities. It occurs in immunocompetent patients without invasion of the mucous membrane on histopathology. 3,7 Aspergillus is the most common agent involved in this pathology, especially Aspergillus fumigatus, but A. flavus or Scedosponum may also be found. The maxillary sinus followed by the sphenoid sinus are the main locations. ${ }^{8}$

Case 3: A 40 year old female came with complaints of left sided nasal obstruction \& headache for more than 3 years. Nasal discharge, loss of sensation of smell was present for last 7 months. She had similar complaints in the past and underwent FESS, 15yrs. before. Nasal obstruction used to get relieved by using nasal spray and headache by taking paracetemol. She started taking both regularly without proper prescription.

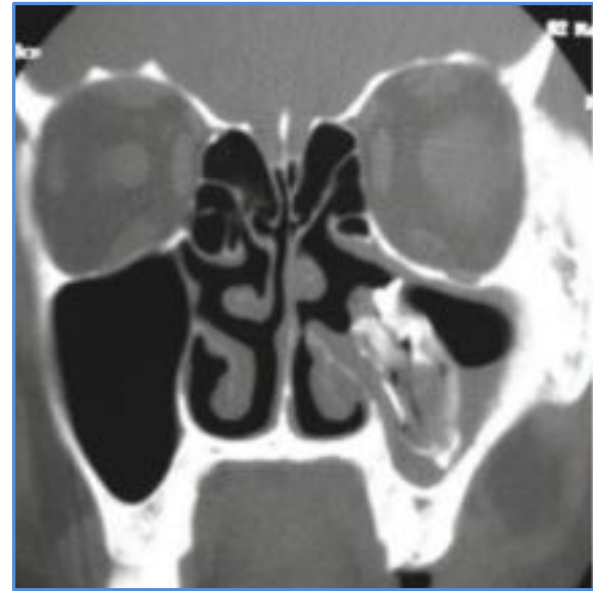

Fig. 5: CT Scan showing fungal ball

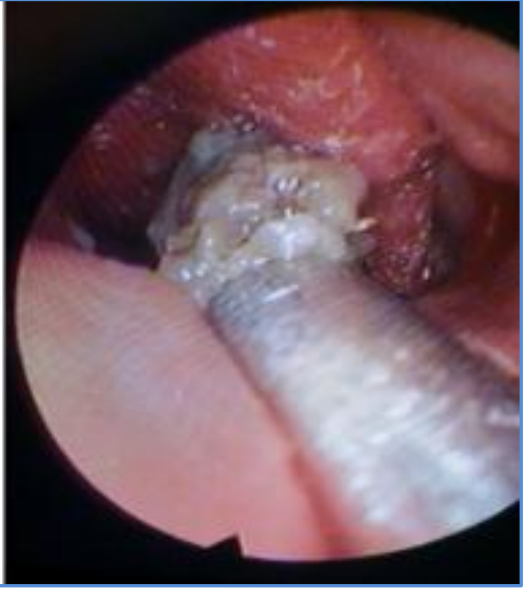

Fig. 6: Endoscopic removal of fungal ball 
Surgical removal of the fungus ball by an endonasal approach under endoscopic guidance was done after enlargement of the natural ostium of the sinus. The fungus ball is aspirated away from the underlying mucous membrane. No medical treatment is required.

Allergic Fungal Sinusitis: Most common form of fungal sinusitis is seen in warm, humid climates. Hypersensitivity reaction to inhaled fungal organisms results in chronic noninfectious inflammatory reaction.

Associated bilateral sinonasal polyposis can be seen"Allergic mucin" within affected sinus which is inspissated mucous the consistency of peanut butter with eosinophils on histology. ${ }^{9}$ Fungi implicated in AFS are known as the dematiaceous (darkly pigmented) fungi-Aspergillus spp, Bipolaris, Curvularia,Alternaria. ${ }^{10}$

Case 4: A 37 year old male came with complaints of bilateral nasal obstruction \& nasal discharge for 10 years. He had associated complaints of dust allergy, recurrent sneezing and watering of eyes. Bilateral greyish multiple polyps and mucinous discharge was seen on anterior rhinoscopy. CT Scan shows hypodense masses filling the nasal cavity, maxillary sinuses and ethmoid sinuses completely. Per operatively greenish brown mucopurulent debris was seen in the maxillary sinus, anterior ethmoidal sinus. The characteristic "Allergic mucin" was seen.

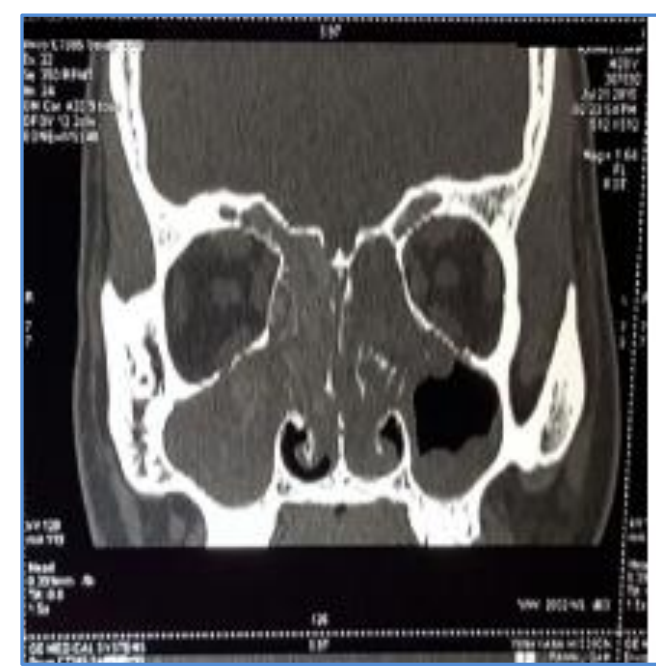

Fig. 7: CT Scan findings

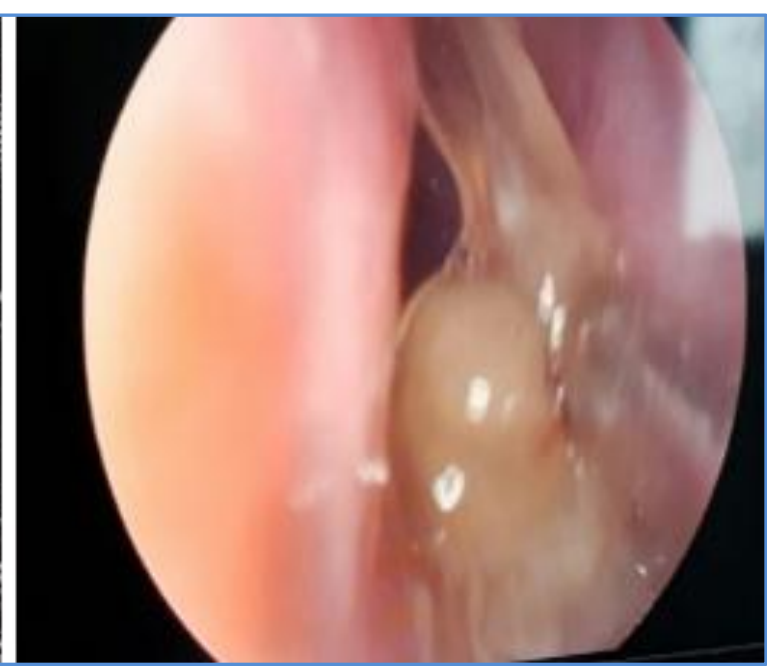

Fig. 8: Allergic Mucin

CONCLUSION: Though the course of sinusitis especially with regards to its complications has been altered by the advent of antibiotics, significant morbidity and mortality is still seen in the developing countries like India. There is an increasing trend among patients and even doctors to consider sinusitis unimportant. This is largely due to ignorance, poverty, and under treatment on the part of the patients and delayed or missed diagnosis on the part of the clinicians. Health education and awareness programmes aimed at understanding the magnitude of the problem and urgent appropriate measures instituted on time become imperative. 


\section{REFERENCES:}

1. Ogunleye AOA, Nwaorgu OGB, Lasisi AO. Complications of sinusitis in Ibadan, Nigeria. West Afr J Med 2001; 20: 98 - 101.

2. Lund VJ. The complications of sinusitis in: Mackay IS, Bull TR (eds). Scott Brown Otolaryngology. Rhinology. Butterworth - Heimann, Edinburgh. 1997; 13/1 - 13/11.

3. DeShazo RD, Chapin K, Swain RE. Fungal sinusitis. New England Journal of Medicine. 1997; 337: 254-9.

4. Hunt SM, Miyamoto RC, Cornelius RS, Tami TA. Invasivefungal sinusitis in the immunodeficiency syndrome. Otolaryngologic Clinics of North America.2000; 33: 335-47.

5. Gillespie MB, O'Malley BW. An algorithmic approach to the management of invasive rhinosinusitis in the immunocompromised patient. Otolaryngologic Clinics of North America. 2000; 33:323-34.

6. Murthy JM, Sundaram C, Prasad VS, Purohit AK, Rammurti S, Laxmi V. Sinocranialaspergillosis: a form of central nervous system Aspergillosis in south India. Mycoses.2001; 44: 141-5.

7. Klossek JM, Serrano E, Peloquin L, Percodani J, Fontanel JP,Pessey JJ. Functional endoscopic sinus surgery and 109 mycetomas of paranasal sinuses. Laryngoscope. 1997; 107: 112-7.

8. Klossek JIVI, Peloquin L, Fourcroy P J, Ferrie JC, Fontanel JP. Aspergillomas of the sphenoid sinus: a series of 10 cases treated by endoscopic sinus surgery. Rhinology. 1996; 34:179-83.

9. Kuhn FA, Javer AR. Allergic fungal rhinosinusitis: our experience. Archives of Otolaryngology Head and Neck Surgery. 1998; 124: 1179-80.

10. Katzenstein AL, Sale SR, Greenberger PA. Allergic Aspergillus sinusitis: a newly recognized form of sinusitis. Journal of Allergy and Clinical Immunology. 1983; 72:89-93.

\section{AUTHORS:}

1. I. Arumugam

2. Jalagandeesh B.

3. Ganesh Bala

4. Ishani Borah

5. Sandeep Jith

\section{PARTICULARS OF CONTRIBUTORS:}

1. Professor and HOD, Department of ENT, Vinayaka Missions Medical College, Karaikal.

2. Post Graduate (Final Year), Department of ENT, Vinayaka Missions Medical College, Karaikal.

3. Assistant Professor, Department of ENT, Vinayaka Missions Medical College, Karaikal.

FINANCIAL OR OTHER COMPETING INTERESTS: None
4. Post Graduate (2 $2^{\text {nd }}$ Year), Department of ENT, Vinayaka Missions Medical College, Karaikal.

5. Post Graduate ( $1^{\text {st }}$ Year), Department of ENT, Vinayaka Missions Medical College, Karaikal.

\section{NAME ADDRESS EMAIL ID OF THE} CORRESPONDING AUTHOR:

Jalagandeesh B, House No. 6, Arumai Nagar, Keezha Kasa Kudy, Kottucherry, P.O., Karaikal-609609, Puducherry.

E-mail: drjoeyblack@yahoo.co.in Date of Submission: 25/08/2015. Date of Peer Review: 26/08/2015. Date of Acceptance: 29/09/2015. Date of Publishing: 08/10/2015. 